\title{
Correction to: A phase I/II study of gemcitabine during radiotherapy in children with newly diagnosed diffuse intrinsic pontine glioma
}

\author{
Sophie E. M. Veldhuijzen van Zanten ${ }^{1}$ - Fatma E. El-Khouly ${ }^{1,2}$ - Marc H. A. Jansen ${ }^{1} \cdot$ Dewi P. Bakker ${ }^{3}$. \\ Esther Sanchez Aliaga ${ }^{4}$. Cornelis J. A. Haasbeek ${ }^{5}$ Nicole I. Wolf ${ }^{3,6}$. C. Michel Zwaan ${ }^{7}$. W. Peter Vandertop ${ }^{8,9}$. \\ Dannis G. van Vuurden ${ }^{1,11} \cdot$ Gertjan J. L. Kaspers ${ }^{1,10}$
}

Published online: 23 October 2017

(c) The Author(s) 2017

\section{Correction to: J Neurooncol DOI 10.1007/s11060-017-2575-9}

In Table 2 of the original publication, there were errors in the baseline scores for the PedsQL TM 3.0 Cancer Module questionnaire, so a corrected version of Table 2 is shown in this erratum. In the subcategories of the PedsQL TM 3.0 Cancer Module questionnaire, nausea and fear of procedure did not score significantly lower after treatment compared to baseline. So, based on the corrected data in Table 2, there was no significant decrease in the total score of the cancer questionnaire, and this statement in the previous manuscript was incorrect.

The online version of the original article can be found under doi:10.1007/s11060-017-2575-9.

Dannis G. van Vuurden dg.vanvuurden@vumc.nl

1 Department of Pediatric Oncology - Hematology, VU University Medical Center, Amsterdam, The Netherlands

2 Department of Clinical Pharmacology \& Pharmacy, VU University Medical Center, Amsterdam, The Netherlands

3 Department of Child Neurology, VU University Medical Center, Amsterdam, The Netherlands

4 Department of Radiology \& Nuclear Medicine, VU University Medical Center, Amsterdam, The Netherlands

5 Department of Radiotherapy, VU University Medical Center, Amsterdam, The Netherlands
6 Neuroscience Amsterdam, Amsterdam, The Netherlands

7 Department of Pediatric Oncology - Hematology, Erasmus MC-Sophia Children's Hospital, Rotterdam, The Netherlands

8 Neurosurgical Center Amsterdam, VU University Medical Center, Amsterdam, The Netherlands

9 Neurosurgical Center Amsterdam, Academic Medical Center, Amsterdam, The Netherlands

10 Princess Máxima Center for Pediatric Oncology, Utrecht, The Netherlands

11 Department of Pediatrics, Division of Oncology Hematology, VU University Medical Center, De Boelelaan 1117, Room 9D36, 1081 HV Amsterdam, The Netherlands 
Table 2 Quality of Life assessment

\begin{tabular}{|c|c|c|c|}
\hline & \multicolumn{2}{|l|}{ Median [IQR] } & \multirow[t]{2}{*}{$P$ value $(<0.05)$} \\
\hline & Week 0 & Week 19 & \\
\hline \multicolumn{4}{|c|}{ PedsQL TM 4.0 Generic Core Scales } \\
\hline \multicolumn{4}{|l|}{ Self-report } \\
\hline Physical performance & 87.50 [37.50;96.88] & $85.94[64.06 ; 93.75]$ & 0.854 \\
\hline Psychosocial health & $85.00[73.33 ; 86.67]$ & $87.50[76.67 ; 93.33]$ & 0.273 \\
\hline Total score & $82.61[60.87 ; 86.96]$ & $84.78[74.46 ; 93.48]$ & 0.715 \\
\hline \multicolumn{4}{|l|}{ Parent report } \\
\hline Physical performance & $78.13[28.13 ; 90.63]$ & $82.81[62.50 ; 86.72]$ & 0.715 \\
\hline Psychosocial health & $44.57[42.39 ; 54.35]$ & $48.91[46.47 ; 49.73]$ & 0.144 \\
\hline Total score & $44.57[42.39 ; 54.35]$ & $48.91[46.47 ; 49.73]$ & 0.144 \\
\hline \multicolumn{4}{|c|}{ PedsQL TM Multidimensional Fatigue Scale } \\
\hline \multicolumn{4}{|c|}{ Self-report } \\
\hline General score & $75.00[58.33 ; 89.58]$ & 83.33 [75.00;91.67] & 0.713 \\
\hline Sleep score & $79.17[56.25 ; 97.92]$ & $83.33[70.83 ; 93.75]$ & 0.414 \\
\hline Cognitive fatigue score & $79.17[66.67 ; 100.00]$ & $83.33[62.50 ; 97.92]$ & 0.713 \\
\hline Total score & $81.94[61.81 ; 88.19]$ & $81.94[73.61 ; 90.97]$ & 0.500 \\
\hline \multicolumn{4}{|l|}{ Parent report } \\
\hline General score & $66.67[50.00 ; 77.08]$ & $87.50[68.75 ; 93.75]$ & 0.273 \\
\hline Sleep score & $83.33[54.17 ; 93.75]$ & $83.33[83.33 ; 95.83]$ & 0.461 \\
\hline Cognitive fatigue score & $75.00[56.25 ; 89.58]$ & $95.83[77.08 ; 97.92]$ & 0.141 \\
\hline Total score & $81.94[54.86 ; 84.72]$ & $86.11[81.11 ; 92.36]$ & 0.080 \\
\hline \multicolumn{4}{|c|}{ PedsQL TM 3.0 Cancer Module } \\
\hline \multicolumn{4}{|c|}{ Self-report } \\
\hline Pain & $100.00[68.75 ; 100.00]$ & $100.00[75.00 ; 100.00]$ & 0.276 \\
\hline Nausea & $85.00[60.00 ; 97.50]$ & $80.00[60.00 ; 90.00]$ & 1.000 \\
\hline Fear of procedures & $83.33[25.00 ; 100.00]$ & $16.67[4.17 ; 50.00]$ & 0.131 \\
\hline Fear of treatment & $100.00[62.50 ; 100.00]$ & $75.00[54.17 ; 100.00]$ & 0.414 \\
\hline Worry & $100.00[66.67 ; 100.00]$ & $83.33[75.00 ; 100.00]$ & 0.655 \\
\hline Cognitive functioning & $80.00[62.50 ; 100.00]$ & $75.00[50.00 ; 100.00]$ & 0.197 \\
\hline Appearance & $100.00[75.00 ; 100.00]$ & $100.00[87.50 ; 100.00]$ & 0.785 \\
\hline Communication & $83.33[70.83 ; 100.00]$ & $83.33[70.83 ; 100.00]$ & 0.157 \\
\hline Total score & $77.78[75.93 ; 90.28]$ & $77.78[69.44 ; 80.56]$ & 0.080 \\
\hline \multicolumn{4}{|l|}{ Parent report } \\
\hline Pain & $87.50[68.75 ; 100.00]$ & $100.00[87.50 ; 100.00]$ & 1.000 \\
\hline Nausea & $90.00[62.50 ; 97.50]$ & $65.00[40.00 ; 85.00]$ & 0.223 \\
\hline Fear of procedures & $75.00[25.00 ; 100.00]$ & $25.00[00.00 ; 58.33]$ & 0.109 \\
\hline Fear of treatment & $75.00[45.83 ; 100.00]$ & $75.00[54.17 ; 100.00]$ & 0.655 \\
\hline Worry & $91.67[62.50 ; 100.00]$ & $75.00[75.00 ; 100.00]$ & 0.854 \\
\hline Cognitive functioning & $65.00[44.38 ; 75.00]$ & $60.00[55.63 ; 75.00]$ & 0.892 \\
\hline Appearance & $83.33[66.67 ; 100.00]$ & 83.33 [75.00;95.83] & 0.577 \\
\hline Communication & $83.33[54.17 ; 100.00]$ & $100.00[87.50 ; 100.00]$ & 0.564 \\
\hline Total score & $78.85[64.35 ; 86.22]$ & $72.22[68.32 ; 81.02]$ & 0.080 \\
\hline
\end{tabular}

